\title{
Abnormal Intraventricular Conduction by ECG Finding
}

National Cancer Institute

\section{Source}

National Cancer Institute. Abnormal Intraventricular Conduction by ECG Finding. NCI

Thesaurus. Code C99543.

An electrocardiographic finding of an atypical passage of electrical impulses through the cardiac ventricles. This includes fascicular blocks (hemiblocks), bundle branch blocks, nonspecific conduction delays and ventricular pacing. (ACC) 